\title{
Enhanced Range Free Localization in Wireless Sensor Networks
}

\author{
Dr. Gaurav Sharma ${ }^{1}$ and Dr. Manjeet Kharub ${ }^{2}$ \\ ${ }^{1}$ Asst. Professor, CVR College of Engineering/ECE Department, Hyderabad, India \\ Email: ergaurav209@yahoo.co.in \\ ${ }^{2}$ Asst. Professor, CVR College of Engineering/ Mechanical Engg. Department, Hyderabad, India \\ Email: manjeetkharub@gmail.com
}

\begin{abstract}
Node localization is one of the most crucial issues for wireless sensor networks (WSNs), as many applications depend on the precise location of the sensor nodes. In last two decades, a number of range-based and range-free localization algorithms have been proposed. Range-free techniques are relatively more efficient but have poor localization accuracy. One of the most widely used range-free techniques is Distance Vector Hop (DV-Hop) algorithm which attracted more attention of researchers due to its stability, feasibility and less hardware cost. To achieve higher accuracy in range free localization algorithms, an Improved DV-Hop algorithm based on Teaching Learning Based Optimization (IDV-Hop based on TLBO) is proposed. In the proposed algorithm, average hop size of the anchor node is modified by updating a correction factor in traditional DV-Hop algorithm. For further improvement of the accuracy, TLBO technique in IDV-Hop is used. With the help of bounded population feasible region, IDV-Hop using TLBO locates the unknown nodes more accurately and achieves higher convergence rate relatively. Simulation results show that the proposed algorithm is efficient and more accurate in terms of localization accuracy compared to DV-Hop, DV-Hop based on Genetic Algorithm (GADV-Hop) and DV-Hop using PSO (Particle Swarm optimization) algorithms.
\end{abstract}

Index Terms: Wireless sensor networks, Localization, DVHop Algorithm, Optimization, TLBO.

\section{INTRODUCTION}

Recent advancements in wireless communication and micro electromechanical systems (MEMS) technology have led to the development of small, smart and cost effective nodes for sensing physical phenomenon known as Sensor Nodes (SNs). These tiny sensor nodes comprise of processor, dedicated memory, transceiver, actuator, sensor(s) and power module [1]. Due to size and cost constraint, SNs have limited power and low computational capabilities. SNs are deployed manually or randomly in the area of interest to monitor the physical phenomenon such as temperature, pressure, sound, velocity, light, humidity, etc. Depending upon the applications, a collection of hundreds or thousands of nodes may be deployed, which are capable of communicating with each other through a wireless medium and form a network called wireless sensor network (WSN). Nodes can sense the desired data, exchange this data among each other, perform some computational tasks on that and transmit this data to a centralized unit called sink node or base station.

WSN has a number of prospective applications in various areas such as surveillance, habitat and environmental monitoring, military applications, healthcare, structural monitoring and disaster management [3]. Among all these, many applications depend on the accurate positions of the nodes to report sensed data meaningful.

Since sensed data without location information is of no use for the user as it is necessary for the user to know the event as well as the location of the event. Manual deployment of nodes is one of the easy ways for location estimation but it is not feasible for a large scale network and in inaccessible areas. Addition of Global Positioning System (GPS) [4] receiver to the node is also another possible way for node localization. But unfortunately, it is not viable for such a large sensor network as it adds up to the size, power consumption and cost of network deployment and having imprecision in non-line of sight (NLOS) environments etc. [6]. One possible solution is to equip only small number of nodes with GPS known as anchor nodes (ANs) or beacon nodes and localize remaining nodes with the help of these anchor nodes through any localization algorithm.

Localization algorithms can be broadly categorized as: range-based and range-free localization. Range-based algorithms need to calculate the distances between ANs and SNs based on the actual range or angle information and use this information to determine the coordinates of unknown nodes [5]. These schemes show good localization accuracy but need an extra hardware for actual range determination and are easily affected by the multipath fading [2]. In contrast, range-free schemes use connectivity information and hop values between $\mathrm{ANs}$ and $\mathrm{SNs}$ to estimate the position of location unaware nodes but show relatively poor localization accuracy. Due to some limitations in rangebased schemes, range-free schemes have attracted worldwide researchers' interest because these are the cost effective alternate solution without any extra range determination hardware.

Distance Vector Hop (DV-Hop) $[6,13]$ is one of the most widely used range-free localization algorithms that has poor localization accuracy which will be discussed thoroughly in the next section. To achieve desired localization accuracy, an improved Distance Vector Hop (IDV-Hop) localization algorithm based on Teaching Learning Based Optimization (TLBO) [10], [11] is proposed in this paper; IDV-TLBO, which is a range free distributed scheme. TLBO is used to improve the localization accuracy of the DV-Hop algorithm.

The remaining paper is structured as follows: Basic DVHop algorithm and some modified DV-Hop algorithms are presented in Section II. In Section III, proposed algorithm (IDV-TLBO) is presented. Then the simulation parameters 
and experimental results are discussed in Section IV. Finally, the whole paper is concluded in Section V.

\section{RELATED WORK}

In literature, a number of range-free localization algorithms have been proposed during last two decades such as Centroid [7], Distance Vector Hop (DV-Hop) [6,13], Approximate Point In Triangle (APIT) [9], Convex Position Estimation (CPE) [8], Multidimensional Scaling (MDS) [12] and many more. But among all these range-free algorithms, DV-Hop has attracted more attention of researchers due to its simplicity, stability, feasibility and less hardware requirement. However, DV-Hop has poor localization accuracy and needs an improvement in it. Basic DV-Hop localization algorithm and some improved versions of DVHop are presented in the next part of this section.

\section{A. DV-Hop Algorithm}

DV-Hop localization algorithm was first proposed by Dragons Niculescu et al. [13]. Many range-free and rangebased localization algorithms work for those unknown nodes which have at least three neighbour anchor nodes for position estimation. But DV-Hop algorithm can also work for those unknown nodes which have few or even no anchor at their neighbour. Since in real time scenarios, anchor nodes are deployed in a very small quantity as compared to normal nodes. Generally, DV-Hop algorithm works into following three steps $[13,14]$ :

Step 1 Estimation of minimum hop counts between every unknown node and anchor nodes.

First of all, every anchor node $A_{i}$ broadcasts a packet containing the location of $A_{i}$ and hop count field starting with 0 . These packets are broadcasted throughout the network. During broadcasting of packets, hop count field is increased by 1 with every hop. That means, on reception of this packet, every node $J$ (here, $J$ can be either normal or anchor node) records the location of $A_{i}$ and initializes $h o p_{i, J}$. Here $h_{o p}, J$ is the minimum hop count between $J$ and $A_{i}$. The value of hop count field varies according to the packets received by anchors with different hop counts. Suppose the received packet is having the lower hop count value than $h o p_{i, J}$, then $J$ will update $h o p_{i}$ according to this lower hop count value, otherwise, $J$ will ignore the packet. By this process, every node gets the minimal hop counts to all anchors in the network.

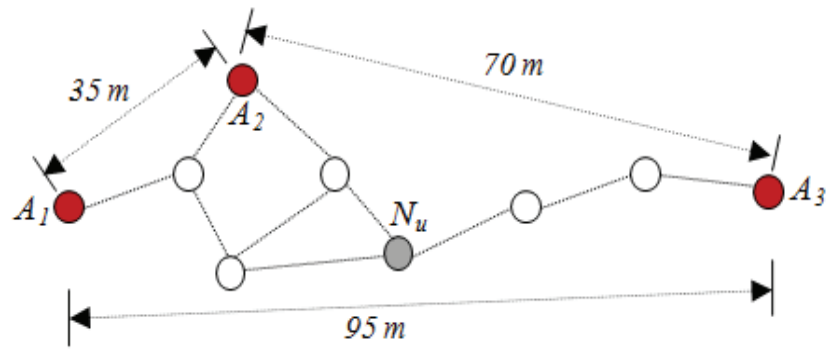

Figure 1. An example of DV-Hop Algorithm
Figure 1 shows an example of a DV-Hop algorithm, in that $A_{1}, A_{2}$ and $A_{3}$ are the anchor nodes and remaining nodes are location unaware nodes. The actual distance between $A_{1}$ and $A_{2}$ is $35 \mathrm{~m}, A_{2}$ and $A_{3}$ is $70 \mathrm{~m}$ and $A_{1}$ and $A_{3}$ is $95 \mathrm{~m}$. Dotted lines show the connectivity between nodes. At a moment, $N_{u}$ is taken as the unknown node to describe the process clearly. From the step 1 of the algorithm, $N_{u}$ has the hop counts 3,2 and 3 to the anchor node $A_{1}, A_{2}$, and $A_{3}$ respectively.

Step 2 Estimation of average hop size of every anchor.

From step 1 , every anchor $A_{i}$ has received the coordinates of other anchors and its minimal hop count values to other anchor nodes. Now $A_{i}$ calculates its average distance per hop or hop size denoted as HopSize $e_{i}$ in the following expression.

HopSize $i=\frac{\sum_{i \neq j} \sqrt{\left(x_{i}-x_{j}\right)^{2}+\left(y_{i}-y_{j}\right)^{2}}}{\sum_{i \neq j} \text { hop }_{i j}}$

where $\left(x_{i}, y_{i}\right)$ and $\left(x_{j}, y_{j}\right)$ are the coordinates of anchor nodes $i$ and $j$ respectively. hop $p_{i j}$ denotes the hop counts between anchor $i$ and $j(i \neq j)$. For example in Figure 1, average distance per hop or hop size of anchors can be calculated as follows:

$$
\begin{aligned}
& \text { HopSize }_{1}\left(A_{1}\right)=(35+95) /(2+6)=16.25 \mathrm{~m} \\
& \text { HopSize }_{2}\left(A_{2}\right)=(35+70) /(2+5)=15 \mathrm{~m} \\
& \text { HopSize }_{3}\left(A_{3}\right)=(95+70) /(5+6)=15 \mathrm{~m}
\end{aligned}
$$

Once average distance per hop is calculated, it is broadcasted through the network by $A_{i}$.

Step 3 Calculation of coordinates of unknown nodes.

After receiving the average distance per hop, a node can calculate the distance $d_{i j}$ from the anchor nodes by multiplying the HopSize $_{i}$ to the hop count from that particular anchor as follows:

$d_{i j}=$ HopSize $_{i} \times$ hop $_{i j} \quad$, where $i \neq j$

For an example in Figure 1, node $N_{u}$ can obtain the approximated distances from each anchor node by formula (2) as: $d_{1}=3 \times 16.25=48.75 m, d_{2}=2 \times 15=30 m, d_{3}=3 \times 15$ $=45 \mathrm{~m}$

After calculating the estimated distances $d_{i}$ from each anchor $A_{i}, i \in\{1,2,3 \ldots \ldots k\}$ where $k$ is the total number of anchors, normal nodes can use trilateration or multilateration techniques to calculate their coordinates. Therefore, the following equations can be derived, where $(x, y)$ is the estimated coordinate of an unknown node.

$$
\left\{\begin{array}{c}
\left(x-x_{1}\right)^{2}+\left(y-y_{1}\right)^{2}=d_{1}^{2} \\
\left(x-x_{2}\right)^{2}+\left(y-y_{2}\right)^{2}=d_{2}^{2} \\
\cdot \\
\cdot \\
\left(x-x_{k}\right)^{2}+\left(y-y_{k}\right)^{2}=d_{k}^{2}
\end{array}\right.
$$


Equation (3) can be expressed as:

$$
\left\{\begin{aligned}
& 2\left(x_{k}-x_{1}\right) x+2\left(y_{k}-y_{1}\right) y=d_{1}^{2}-d_{k}^{2}-x_{1}^{2}+x_{k}^{2}-y_{1}^{2}+y_{k}^{2} \\
& 2\left(x_{k}-x_{2}\right) x+2\left(y_{k}-y_{2}\right) y=d_{2}^{2}-d_{k}^{2}-x_{2}^{2}+x_{k}^{2}-y_{2}^{2}+y_{k}^{2} \\
& \cdot \\
& 2\left(x_{k-1}-x_{k}\right) x+2\left(y_{k-1}-y_{k}\right) y \cdot d_{k-1}^{2}-d_{k}^{2}-x_{k-1}^{2}+x_{k}^{2}-y_{k-1}^{2}+y_{k}^{2} \\
& \hline
\end{aligned}\right.
$$

Equation (4) can be expressed in the linear form as:

$A X=B$; where

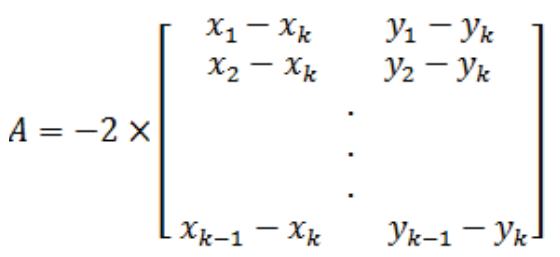

$B=\left[\begin{array}{c}d_{1}^{2}-d_{k}^{2}-x_{1}^{2}+x_{k}^{2}-y_{1}^{2}+y_{k}^{2} \\ d_{2}^{2}-d_{k}^{2}-x_{2}^{2}+x_{k}^{2}-y_{2}^{2}+y_{k}^{2} \\ \cdot \\ \cdot \\ d_{k-1}^{2}-d_{k}^{2}-x_{k-1}^{2}+x_{k}^{2}-y_{k-1}^{2}+y_{k}^{2}\end{array}\right]$

$X=\left[\begin{array}{l}x \\ y\end{array}\right]$

After solving equation (4) based on least square estimations; unknown node can obtain its estimated coordinate as follows:

$X=\left(A^{T} A\right)^{-1} A^{T} B$

where $A^{T}$ is the transpose of the matrix $A$, and $A^{-1}$ is the inverse of matrix $A$.

Since the DV-Hop algorithm is simple to implement and can localize the nodes which have a few or even no neighbour anchors but its localization accuracy needs to be improved. Many improved versions of DV-Hop algorithm have been proposed in the literature, some of them are discussed here.

An improved DV-Hop algorithm is proposed for WSNs by Dengyi et al. [18]. In this, localization accuracy is improved by two methods throughout the whole process. Firstly, normal node refines the average distance per hop by taking the mean of anchor node's hop size. Secondly, the anchor nodes also refine the average distance per hop with the angle information between normal and anchor node. Calculating angles between normal and anchor nodes make this algorithm more complicated and energy inefficient.

Chen et al. [17] proposed an improved DV-Hop algorithm based on Particle Swarm Optimization (PSO). There are mainly four steps to describe the algorithm. In step 1, few anchors are deployed on the boundary of the sensing field. In step 2, anchors refine the average distance per hop of themselves and broadcast this throughout the whole network. In step 3, distance is calculated by normal nodes and position is estimated using 2-D hyperbolic location algorithm [16].

Finally in step 4, PSO is used to enhance the accuracy of the algorithm. However, anchor nodes placement at the boundary of the sensing field is not a feasible for inaccessible terrain area.

Bo Peng et al. [15] proposed genetic algorithm (GA) based DV-Hop algorithm. The whole algorithm has six steps to perform in which starting three steps are same as for traditional DV-Hop algorithm and last three steps are for the process of genetic algorithm. Though GADV-Hop algorithm improves the localization accuracy up to an extent but by using genetic algorithm, there are some control parameters (crossover, selection and mutation) to be tuned correctly for getting better results.

From the above review, it can be concluded that localization is the optimization problem and its overall estimation error needs to be minimized. This encourages us to propose an Improved DV-Hop algorithm based on Teaching Learning Based Optimization (TLBO). The full process of TLBO is given in $[10,11]$.

\section{Proposed ALGORITHM}

It is well known that DV-Hop algorithm is based on Distance Vector (DV) routing. The distance between the nodes is estimated by the hop count value multiplied by the hop size of the anchor. But there is a large amount of error between the calculated and actual value when there are two or more hop counts between anchor node and normal node. The deviation created by anchors is not considered in the typical DV-Hop algorithm. The improved algorithm implements the revised hop size in the network to calculate the distance between the normal node and anchor node and uses the teaching learning based optimization (TLBO) to estimate the correct location of the nodes.

In the proposed algorithm, starting two steps are same as described in DV-Hop algorithm of section 2. In step 3, calculated distance is modified. Consider $D_{i j}$ is the distance between two anchors $i$ and $j$, calculated according to equation (2), as follows:

$D_{i j}=$ HopSize $_{i} \times$ hop $_{i j}$, where $i \neq j$

The actual distance between two anchor nodes can be calculated by their coordinates, as follows:

$D_{i j}^{r}=\sqrt{\left(x_{i}-x_{j}\right)^{2}+\left(y_{i}-y_{j}\right)^{2}}$, where $i \neq j$

where $\left(x_{i}, y_{i}\right)$ and $\left(x_{j}, y_{j}\right)$ are the coordinates of anchors $i$ and $j$ respectively. Now from equation (9) and (10), difference between the estimated distance and actual distance; $d_{i j}^{e}$ (distance error) can be calculated by the following relationship.

$d_{i j}^{e}=\left|D_{i j}-D_{i j}^{r}\right|$

Now this distance error value is used to modify the average distance per hop value of ANs by adding the 
correction factor $\psi$ to the previous hop size value of the particular anchor. This correction factor is used to refine the average distance per hop deviation of the anchor node. $\psi_{i}$ is the correction factor of an anchor $i$ and can be calculated using formula (12).

$\psi_{i}=\frac{\sum_{i \neq j} d_{i j}^{e}}{\sum_{i \neq j} h o p_{i j}}$

Finally the particular distance between normal node and anchor node can be determined by adding the correction factor into the average hop size of the anchor and multiplied by the hop counts between normal node and that particular anchor node as shown in the formula (13), which is the modification of formula (2).

$d^{\prime}{ }_{i j}=\left(\right.$ HopSize $\left._{i}+\psi_{i}\right) \times$ hop $_{i j} \quad$, where $i \neq j$

Still the modified distance value calculated from (13) is not so much accurate because it is a calculated value, there may be error. For that reason, a very efficient optimization technique viz. TLBO is used to improve the localization accuracy in WSN. Therefore, the localization problem can be expressed in terms of objective function as:

$$
f\left(x_{u}, y_{u}\right)=\operatorname{Min}\left(\sum_{\substack{i=1,2 \ldots k \\ u=k+1 \ldots \ldots, n}}\left|\sqrt{\left(x_{u}-x_{i}\right)^{2}+\left(y_{u}-y_{i}\right)^{2}}-d_{i u}\right|\right)
$$

where $\left(x_{i}, y_{i}\right)$ is the coordinates of anchor nodes, $i=1,2 \ldots k$. $\left(x_{u}, y_{u}\right)$ is the estimated coordinates of unknown nodes, $u=k+1, k+2 \ldots . n$ and $d_{i u}$ is the distance between anchor nodes and normal nodes, calculated according to formula (13). There are total $N$ sensor nodes in the network i.e. $N=k+n$.

Although TLBO is free from the algorithm parameters unlike other optimization techniques yet creation of initial population is necessary for the further process.

\section{Simulation PARAMETER AND RESUlts}

In this section, the simulation of proposed algorithm viz. IDV-Hop using TLBO in MATLAB 7.10 is presented to evaluate the performance in terms of localization errors with different parameters and also make comparisons with some other existing algorithms viz. DV-Hop [13], GADV-Hop [15] and DV-Hop based on PSO [17] under the same scenario.

In the simulations, 100 sensor nodes (anchor nodes $(k)$ and unknown nodes $(n)$ ) are distributed randomly in twodimensional fixed square area of $100 \times 100 \mathrm{~m}^{2}$ as shown in Figure 2, where anchor nodes and unknown nodes are represented by red pentacles and black dots respectively. Communication radius of each node is set to be $25 \mathrm{~m}$. Simulation parameters are shown in Table I.

In TLBO, there is no need to set any algorithm parameters since it is free from these parameters. Therefore, the convergence rate of TLBO is higher than other optimization algorithms [19], as it is achieved the best solution in 15.02 seconds. Convergence curve for the proposed algorithm is shown in Figure 3.

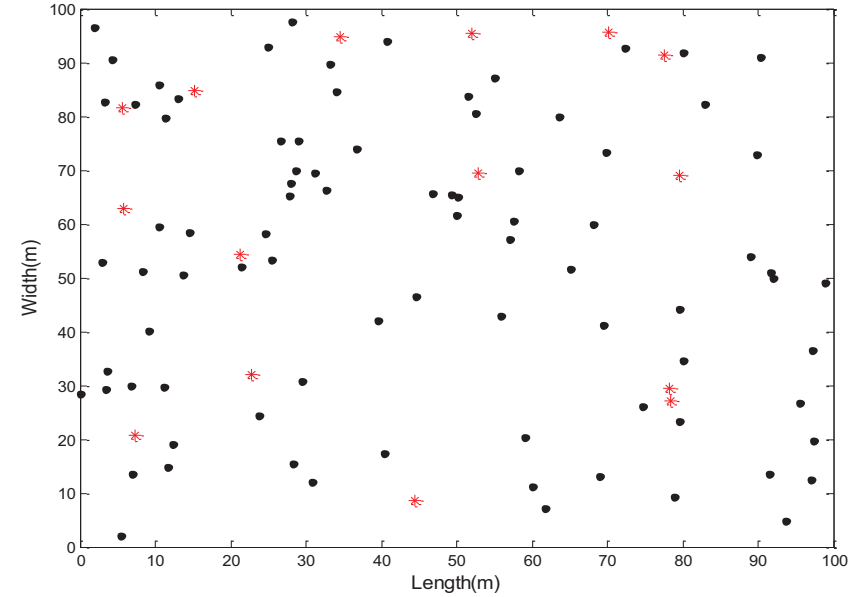

Figure 2. Nodes Distribution

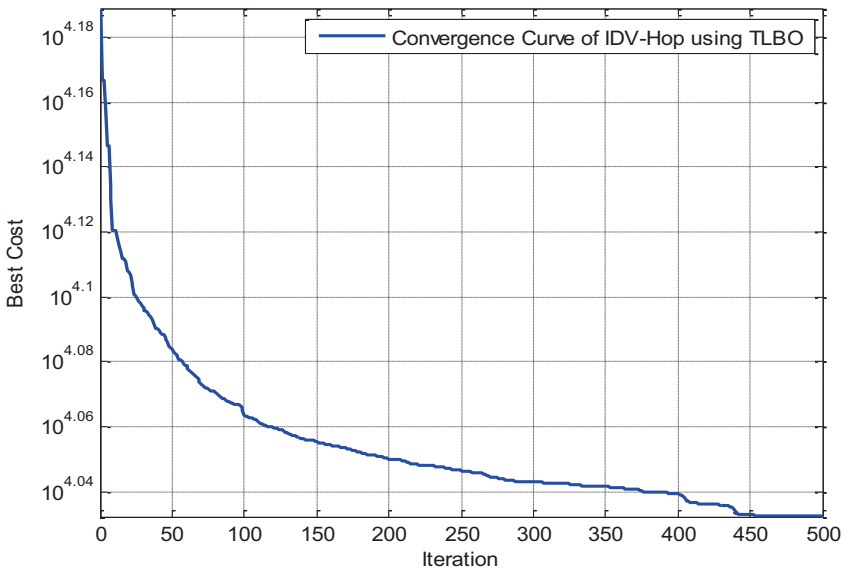

Figure 3. Convergence Curve of proposed algorithm for single simulation run

TABLE I.

SIMULATION PARAMETERS

\begin{tabular}{|l|c|}
\hline \multicolumn{1}{|c|}{ Parameters } & Values \\
\hline Total number of nodes & 100 \\
\hline Area & $100 \times 100 \mathrm{~m}^{2}$ \\
\hline Communication Range & $25 \mathrm{~m}$ \\
\hline Number of anchor nodes & $15 \%$ \\
\hline Initial Population & 50 \\
\hline Maximum Iterations & 500 \\
\hline
\end{tabular}

It needs to set the optimum value of initial population because there are not any specified rules or formulas to set it. As there is a trade-off between small and large population size because it is considered that small population size leads the algorithm to poor solutions and large population size makes the algorithm to expend more computation time to find the optimum solution [32]. When there is no information regarding a possible solution, then it could expect, that the more diverse the initial population is, the greater the possibility to find an optimum solution is. So, initial population is taken as 50 after 30 simulation runs to find the optimum value of it by considering above factors. From Figure 3, it can be seen that best solution is achieved in around 450 iterations; therefore, 500 iterations are taken throughout the whole experiments. As Figure 3 shows the convergence curve of the proposed algorithm for single simulation run at any instant. 


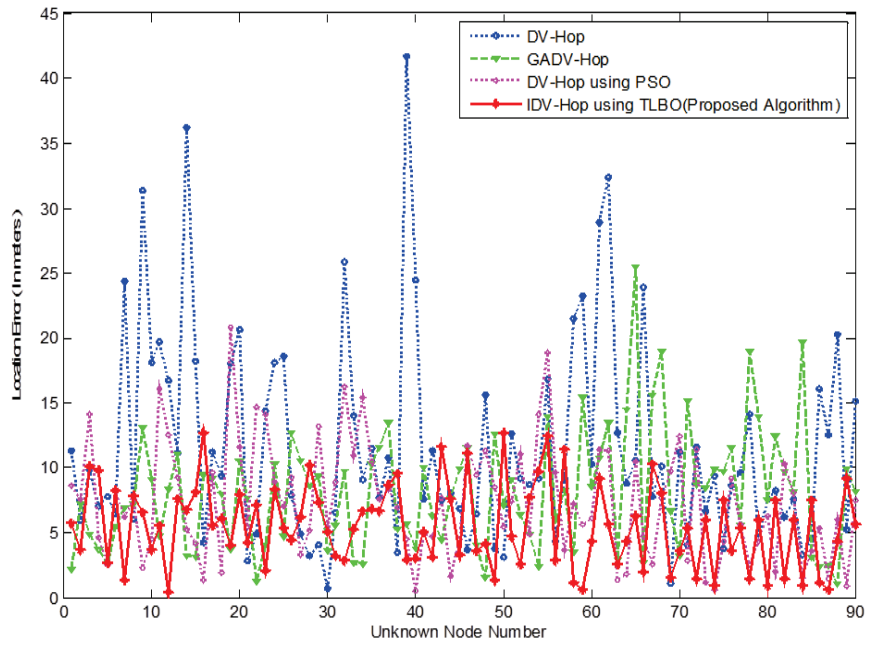

Figure 4. Localization error of each unknown node of single simulation run at any instant $(\mathrm{R}=25 \mathrm{~m}$, Anchor Node Ratio=10\%)

To compare the performance of proposed algorithm with other existing algorithms, the localization error is calculated of each normal node; mathematically it can be expressed by the following formula:

Localization Error $=\sqrt{\left(x_{u}-x_{a}\right)^{2}+\left(y_{u}-y_{a}\right)^{2}}$

where $\left(x_{u}, y_{u}\right)$ is the estimated coordinate and $\left(x_{a}, y_{a}\right)$ is the actual coordinate of the unknown node.

The estimated localization error shows the measure of accuracy of the algorithm. Accuracy is better when the estimation error is smaller. Figure 4 shows the location error of each unknown node of four algorithms at communication radius of $25 \mathrm{~m}$ and when anchor node ratio is $10 \%$ in the network. As shown in Figure 4, proposed algorithm is more stable comparatively and has lower localization error than other three variants of DV-Hop algorithms.

To compare the performance of proposed algorithm with existing techniques, the mean error is calculated of each algorithm with different parameters under the same scenario. Mean error can be determined as the ratio of total localization error to the number of unknown nodes (n) and can be expressed as follows:

$$
\text { Mean Error }=\frac{\sum_{\mathrm{g}, \mathrm{u}=1}^{\mathrm{n}} \sqrt{\left(\mathrm{x}_{\mathrm{u}}-\mathrm{x}_{\mathrm{g}}\right)^{2}+\left(\mathrm{y}_{\mathrm{u}}-\mathrm{y}_{\mathrm{a}}\right)^{2}}}{\mathrm{n}}
$$

Figure 5 shows the estimated localization mean error of these algorithms with a different number of anchor nodes deployed. It can be seen from the results that proposed algorithm performs better than existing three algorithms of [13], [15] and [17]. It is observed from the Figure 5 that when the number of anchor nodes increases, mean localization error of four algorithms decreases.

This is due to the reasons that minimum hop count value between the unknown nodes and anchor nodes decreases with increasing the number of anchor nodes. Also, for accurate hop size of an anchor, more accurate distance between normal node and anchor node is obtained and hence localization error decreases.
The result data of each algorithm is taken as the average of 200 independent simulation experiments.

Figure 6 shows the mean localization error with different communication ranges of the sensor nodes. The communication radius of the sensor nodes is tuned from 15 to $45 \mathrm{~m}$ and ratio of anchor nodes is taken $15 \%$ in the network of 100 sensor nodes. It is observed from the Figure 6 that after the communication range of $25 \mathrm{~m}$, the mean localization error increases gradually of all the algorithms. It is due to the reasons that with the increase of communication range, average hop size of anchor nodes also increases and estimated distance between unknown nodes and anchor nodes also increases, thus affecting the localization accuracy of all algorithms.

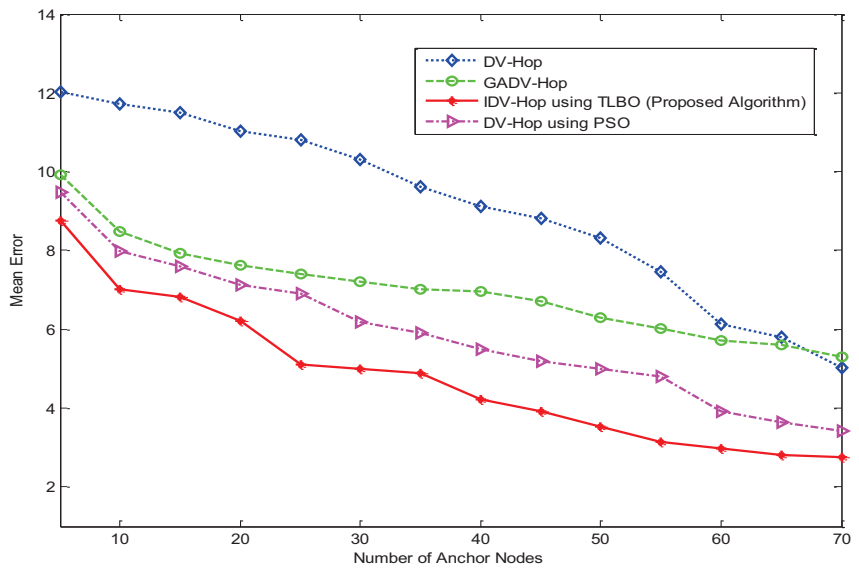

Figure 5. Mean localization error with a different number of anchor nodes.

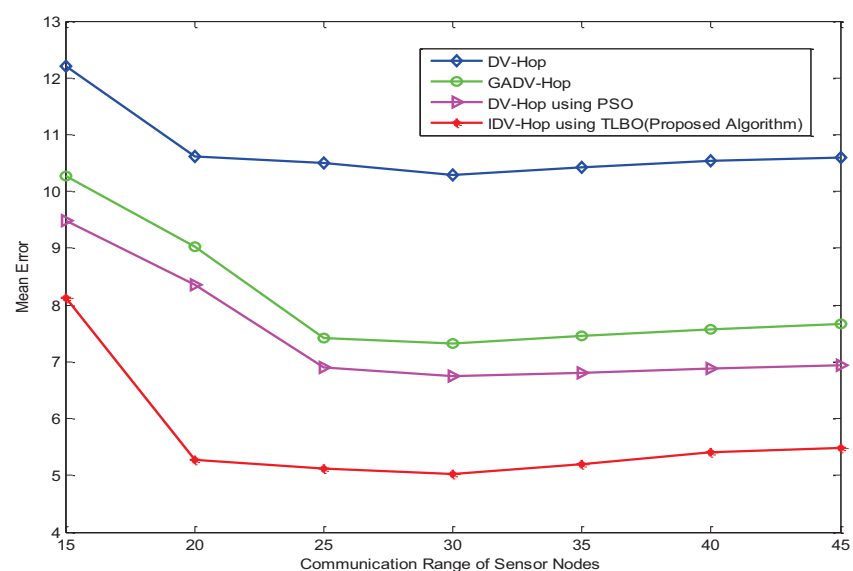

Figure 6. Mean Localization error with the change of communication range of sensor nodes

It can be seen from the simulation results of Figures 4, 5, and 6 , the proposed algorithm viz. IDV-Hop using TLBO outperforms than some existing three algorithms of [13], [15] and [17]. The localization error of the proposed algorithm is smaller than these three algorithms with different parameters (variation of anchor nodes, communication radius, and node density) under the same scenario.

\section{CONCLUSions}

Localization is a crucial issue in WSNs, because various applications depend on the accurate localization of the 
nodes. To minimize the localization errors of the range-free algorithms, IDV-Hop using TLBO technique for WSNs is proposed. In proposed scheme, average hop size of an anchor is modified using correction factor and the distance between the unknown nodes and anchors is calculated based on modified hop size. This calculated distance is closer to the actual distance. Since localization is an optimization problem, which needs to be minimized the localization errors in WSN. To achieve this, an efficient optimization technique viz. TLBO is implemented in this paper, which is free from the algorithms parameters. The proposed algorithm bounds the feasible region of initially generated population. With the help of bounded population feasible region, IDV-Hop using TLBO locates the unknown nodes more accurately and achieves higher convergence rate relatively. Simulation results show that the proposed algorithm has better accuracy as compared to DV-Hop, GADV-Hop, and DV-Hop using PSO algorithms.

\section{REFERENCES}

[1] I. F. Akyildiz, W. Su, Y. Sankarasubramaniam, and E.E Cayirci, "A survey on sensor networks," IEEE Communications Magazine, Vol. 40, no. 8, pp.102-105, 2002.

[2] Boukerchie, A., Oliveria, H. A. B. F., Nakamura, E. F. \& Loureiro, A. A., "Localization systems for wireless sensor networks," IEEE Wireless Communications, Vol. 14, no. 6, pp. 6-12, 2007.

[3] K. Romer and Mattern, "The design space of wireless sensor network" IEEE Trans. Wireless Communications, Vol. 11, no. 6 , pp. 54-61, 2004.

[4] Wellenhof, B.H., Lichtenegger, H., Collins,J. "Global Positioning System: Theory and Practice". Springer-Verlag, 1997.

[5] Zheng, J.,Wu, C., Chu, H., \& Xu, Y. "An improved RSSI measurement in wireless sensor networks" In:2011 Procedia Engineering (Elsevier), vol. 15, pp. 876-880, 2011.

[6] D. Niculescu and B. Nath "Ad Hoc Positioning System (APS)" In: 2001 Proc. Global Telecomm. Conference (Globecom '01), vol. 1, pp. 292 2931, 2001.

[7] N. Bulusu, J. Heidemann, and D. Estrin,"GPS-Less Low Cost Outdoor Localization for Very Small Devices" IEEE Personal Comm. Magazine, vol. 7, no. 5, pp. 28-34, 2000.

[8] L. Doherty, K.S.J. Pister, and L.E. Ghaoui "Convex Position Estimation in Wireless Sensor Networks.” In: 2001 Proc. IEEE INFOCOM '01, vol. 3, pp. 1655-1663, 2001.
[9] G. Sharma and A. Kumar, "Modified Energy-Efficient Range-Free Localization Using Teaching-Learning-Based Optimization for Wireless Sensor Networks," IETE Journal of Research, vol. 64, no. 1, pp. 124-138, Jul. 2017.

[10] R.V. Rao, V.J. Savsani, D.P. Vakharia (2011) "Teachinglearning-based optimization: a novel method for constrained mechanical design optimization problems", Computer-Aided Design, Vol. 43, no. 3, pp. 303-315, 2011.

[11] R.V. Rao, V.J. Savsani, D.P. Vakharia, "Teaching-learningbased optimization: an optimization method for continuous non-linear large scale problems", Information Sciences, Vol. 183, no. 1, pp. 1-15, 2012.

[12] G. Sharma, \& A. Kumar, "Dynamic Range Normal Bisector Localization Algorithm for Wireless Sensor Networks". Wireless Personal Communications, vol. 9, no. 3, pp. 45294549, 2017.

[13] Niculescu, D., \& Nath, B., "DV based positioning in ad hoc networks". Telecommunication Systems, Vol. 22, no. 4, pp. 267-280, 2003.

[14] Gzara, F., \& Erkut, E. "Telecommunications network design with multiple technologies". Telecommunication Systems, Vol. 46, no. 2, 2011.

[15] Bo Peng, Lei Li, "An improved localization algorithm based on genetic algorithm in wireless sensor networks", Cogn Neurodyn, Springer Vol. 9, pp.249-256, 2015.

[16] Sharma, G., \& Kumar, A. "Improved range-free localization for three-dimensional wireless sensor networks using genetic algorithm" Computers \& Electrical Engineering. Vol. 72, no. 11, pp. 808-827, Nov. 2018.

[17] Chen, X., \& Zhang, B., "Improved DV-Hop node localization algorithm in wireless sensor networks" International Journal of Distributed Sensor Networks.,2012 doi:10.1155/2012/ 213980 .

[18] G. Sharma and A. Kumar, "Fuzzy logic based 3D localization in wireless sensor networks using invasive weed and bacterial foraging optimization," Telecommunication Systems, vol. 67, no. 2, pp. 149-162, May 2017.

[19] Lin, J., Liu, H., \& Li, G. "Study for improved DV-Hop localization algorithm in WSN", Application Research of Computers, Vol. 26, no.4, pp. 1272-1274, 2009.

[20] G. Sharma, \& A. Kumar, "Improved DV-Hop localization algorithm using teaching learning based optimization for wireless sensor networks". Telecommunication Systems, vol. 67, no. 2, pp. 163-178, 2017. 